\title{
High-Resolution 3D Fluorescent Imaging of Intact Tissues.
}

Danny El-Nachef $f^{1,2,3,5}$, Amy M. Martinson ${ }^{1,3,5}$, Xiulan Yang ${ }^{1,3,5}$, Charles E. Murry ${ }^{1,2,3,4,5}$, W. Robb MacLellan ${ }^{1,2,5}$

${ }^{1}$ The Institute for Stem Cell and Regenerative Medicine, University of Washington, Seattle, WA 98109, USA

${ }^{2}$ Department of Medicine, Cardiology, University of Washington, Seattle, WA 98195, USA

${ }^{3}$ Department of Pathology, University of Washington, Seattle, WA 98109, USA

${ }^{4}$ Department of Bioengineering, University of Washington, Seattle, WA 98105, USA

${ }^{5}$ Center for Cardiovascular Biology, University of Washington, Seattle, WA 98109, USA

Correspondence: WRMacLellan@cardiology.washington.edu; (206) 616-1040. 


\begin{abstract}
Histological analysis of fluorescently labeled tissues has been a critical tool to understand molecular organization in situ. However, assessing molecular structures within large cells and in the context of human organ anatomy has been challenging because it requires penetration of staining reagents and light deep into opaque tissues, while also conforming to the spatial constraints of high-resolution objective lenses. This methodology article describes optimized sample preparation for sub-micron resolution 3D imaging in human and rodent tissues, yielding imaging depth $(>100 \mu \mathrm{m})$ and resolution $\left(<0.012 \mu \mathrm{m}^{3}\right.$ voxel size $)$ that has previously been limited to whole-mount in vitro organoid systems, embryos, and small model organisms. Confocal images of adult human and rodent organs, including heart, kidney, and liver, were generated for several chemical and antibody stains in cleared tissue sections $>100 \mu \mathrm{m}$ thick. This method can be readily adopted by any lab performing routine histology and takes 3 days from the start of tissue preparation to $3 \mathrm{D}$ images.
\end{abstract}




\section{Introduction}

Analysis of tissue sections has been essential to our understanding of the molecular and cellular mechanisms that control structure and function in mammalian tissues and organs. In fact, the most common histological protocols such as hematoxylin staining of formalin fixed paraffinembedded microtome sections have changed little since their development in the 1800's'. The ability to generate and analyze thin tissue sections (i.e. $<10 \mu \mathrm{m}$ thick) in situ broadly transformed the field because it enabled minimal light scattering, efficient staining reagent penetration, and compatibility with high resolution objective lenses that have small working distances. While fluorescent molecular labeling and imaging modalities have continued to improve resolution of fine structures in 3D, the ability to study these structures within the context of cells or anatomical features that are $>10 \mu \mathrm{m}$ large is limited by light scattering, staining reagent penetration, and highresolution objective lens working distances ${ }^{2}$. Several tissue clearing approaches have overcome light scattering, but the latter limitations remain².

Tissue-clearing protocols that have attempted to address light scattering and penetration limits were recently reviewed ${ }^{2,3}$. While most of these approaches were restricted to embryos and small animal models, three studies demonstrated tissue clearing in human brain or prostate samples ${ }^{4-6}$, though it is unknown how these protocols perform in other human organs. The major limitation shared by all previous reports is that the cleared samples were not imageable with high resolution objectives ${ }^{6-8}$. For the samples to physically fit within the working distance of the objective lens, low powered objectives (i.e. 20x dry, 0.6 numerical aperture (NA)) with large working distances $(>500 \mu \mathrm{m})$ were used. These low NA objectives have limited ability to resolve fine structures because the resolution limit of an objective lens is determined by its NA ${ }^{7,9}$. Abbe's law defines the lateral $(X Y)$ resolution limit as $N / 2 N A$; while axial $(X Z$ and $Y Z$ ) resolution is defined by $2 \lambda /\left(N A^{2}\right)$. Thus, objectives with lower NA have poorer lateral resolution and exponentially worse axial resolution. Recently designed objectives have been developed for cleared tissues that have large working distances and improved NA, but these require custom manufacturing and only modestly improve the NA to $0.9^{10}$. Artificial expansion of specimens can improve spatial resolution with standard large working distance, low NA objectives, but this requires crosslinking proteins to expandable hydrogels ${ }^{8}$, which can introduce distortions from uneven expansion ${ }^{11}$ and dramatically increases sample preparation and imaging time. All reported tissue clearing protocols, even those that do not require expansion microscopy techniques, have practical limitations because the procedure can take several weeks. For example, the Advanced CLARITY protocol (based on CLARITY) takes 7-28 days to perform ${ }^{7}$ and the iDISCO protocol (based on 
3DISCO) takes 8-18 days ${ }^{12}$. Furthermore, many of these studies were restricted to examination of endogenously encoded fluorescent proteins and chemical stains, as antibody-based stains penetrate less efficiently ${ }^{13-15}$. While these approaches can decrease sample preparation time, they require the generation of transgenic lines for each molecule to be studied ${ }^{13}$. In addition, studies in cleared tissues have frequently utilized two-photon excitation fluorescence microscopy to fully utilize the working distance of low resolution objectives, extending imaging depth to several millimeters; but this requires costly, specialized equipment, and has limitations for multichannel acquisitions $^{16,17}$. For instance, DNA dyes and fluorescent proteins that can normally be distinguished by excitation wavelength in single photon microscopy demonstrate overlap with twophoton excitation ${ }^{16-18}$. Another practical consideration is clearing protocols often utilize customized electrophoresis chambers ${ }^{7}$ or proprietary reagents ${ }^{2,19}$ that can be inaccessible or prohibitively expensive for analysis of large specimens. Recently, a protocol was established to clear and image organoids, enabling high resolution 3D imaging of human tissues ${ }^{20}$ but these engineered tissue constructs are much smaller in scale compared to adult mammalian organs and may not completely reflect matured cells within adult tissues in vivo. Advances in micro computed tomography have enabled imaging of thick human specimens, but this modality is not compatible with detection of fluorescent labels and cannot achieve the same resolution as optical approaches $^{21,22}$. While electron microscopy approaches have superior resolution, they are not suitable for 3D imaging in thick specimens ${ }^{7}$.

These challenges prompted us to develop a sample preparation and imaging technique that generates 100-200 $\mu$ m thick sections in suspension, enabling rapid staining and clearing of samples that are compatible with standard high-resolution objectives (i.e. $60 x$ oil, $N A=1.4$ ) and single photon confocal microscopes. These features yielded submicron resolution in all three dimensions within thick tissues that could be imaged in their entirety. Our use of defined, inexpensive clearing reagents and common lab equipment makes this approach accessible to any lab that performs routine histology. Together, this 3-day technique enables assessment of molecular structures in a variety of thick human biopsies and organs, such as cardiac myocyte sarcomeres, gap junctions, and T-tubules, hepatocyte canaliculi, kidney podocyte membranes, capillary networks, and subnuclear chromatin domains. 


\section{Results}

Development of the novel histology plus clearing technique for submicron resolution in 3D

Empowering immunolabeling and high resolution imaging techniques in thick tissues has special relevance to cardiology and studies of cardiac myocytes (CMs) which are $>100 \mu \mathrm{m}$ in $\mathrm{size}^{23}$. The inability to resolve small macromolecules, such as Aurora Kinase B localized to cytokinesis cleavage furrows or condensed chromatin labeled with mitosis marker phosphorylated histone $\mathrm{H} 3$, in whole CMs in situ has led to contentious views on the capacity of adult mammalian CMs to proliferate ${ }^{24-26}$. Dissociation of the heart into single cells and subsequent in vitro analysis may bias against cells sensitive to protease digestion and might not reflect $\mathrm{CM}$ behaviors in vivo ${ }^{27}$, and identification of which cell types are expressing cell proliferation markers in situ has been equivocal using current histology methods that fail to capture CMs in their entirety or clearly resolve individual cells within the densely packed myocardium ${ }^{24}$. Thus, a sample preparation and imaging technique that provides volumetric quantifications and resolves sarcomere structure, chromatin organization, nuclei number, and cell cycle activity in situ in whole adult mammalian tissue holds great promise to address these intractable problems.

To this end, we used protocols we established for whole-mount high resolution 3D imaging of cleared embryos ${ }^{28,29}$ as a starting point. Tissue fixation, embedding, sectioning, staining, optical clearing, and imaging protocols were tested to optimize a procedure in adult mammalian tissues for generating thick, optically clear specimens that could be imaged with sub-micron resolution in $3 \mathrm{D}$. We found that lightly crosslinking the samples and post-fixation treatment with methanol was critical to maintain tissue integrity and for efficient downstream tissue clearing (Figure 1a). Agarose embedding, vibratome sectioning, and benzyl-alcohol benzyl-benzoate (BABB) solubilization effectively generated $>100 \mu \mathrm{m}$ thick, optically clear samples (Figure 1a) that could be imaged in their entirety with high-powered objective lenses and standard singlephoton confocal microscopy.

Prior to 3D imaging the tissue samples, fluorescent beads manufactured with known diameters were examined using the same slide preparation and BABB mounting media as for the tissues to ensure lack of spherical aberrations, z-stretching, or z-compaction (Figure 1b). Normal adult human heart tissues were processed and stained for several markers, and Zintensity profiles were plotted, demonstrating efficient clearing, even staining through the tissue depth, and acceptable levels of photobleaching (Figure 1c). Visualization of 3D reconstructions provided a qualitative assessment of the method's performance (Figure 1d). 
Definitive assessment of cell cycling and chromatin in intact human and mice cardiac myocytes

To assess the proliferative capacity of human adult CMs we subjected normal and cardiomyopathy specimens to this technique and immunostained for a chromatin marker of mitosis, phosphorylated histone $\mathrm{H} 3$ ( $\mathrm{pH} 3$ ). We found no evidence of adult CM proliferation in normal (Figure 2a and Videos 1 and 2) or cardiomyopathy (Figure 2b) human samples, nor in wildtype mice (Figure 2c and Video 3), though mitosis marker pH3 was unequivocally detected in cardiac myocyte nuclei from transgenic mice where the Myc oncogene is specifically induced in adult $\mathrm{CMs}^{30}$ (Figure 2d and Video 4). Importantly, the images demonstrated this method can be used to unequivocally assign nuclei as belonging to CMs or non-CM cell types, and could resolve fine molecular structures, such as individual sarcomeres, T-tubules, and capillaries, in all three dimensions within whole CMs in thick cardiac tissues. Because each z-stack is composed of hundreds of serial images that can take over an hour to acquire on a standard single-photon confocal, we tested this sample preparation with spinning disk confocal microscopy (Figure 2e). This demonstrated comparable 3D reconstructions could be generated in one tenth of the time as standard confocal microscopes and that the sample preparation method is compatible with multiple imaging modalities.

Since epigenetic modifications on chromatin and nuclear morphology have been identified as key mediators of CM proliferation and cardiomyopathies ${ }^{23,31-33}$, we tested whether these features could be resolved by our technique. Nuclear morphology and subnuclear heterochromatin domains that stain positive for canonical heterochromatin markers Heterochromatin Protein 1 (HP1) and histone H3 Lysine 9 trimethylation (H3K9me3) ${ }^{23}$, were clearly resolved in 3D (Figure 3).

Engrafted human pluripotent stem cell-derived cardiac cells partially integrate with host hearts

We next tested whether this method could enable assessment of cardiac cell therapy engraftment and integration with host myocardium at the molecular level in 3D. GFP-labeled human pluripotent stem cell-derived cardiac myocytes were injected into immune-compromised host rat myocardium as previously described ${ }^{34,35}$ (Figure 4). The cleared thick sections were visualized with low-powered objectives to examine the total graft size in relation to total host myocardium, and with high-powered objectives that could resolve sarcomeres in the engrafted myocytes (Figures $\mathbf{4 a}$ and $\mathbf{4 b}$ ). As expected, no GFP+ cells were detected in vehicle injected rat hearts, and Connexin 43+ gap junctions in the rat myocardium were resolvable in 3D (Figure 4c and Video 5). Analysis of cell-injected animals revealed Connexin 43+ gap junctions connecting the engrafted cells and host myocardium 6-weeks after cell transplantation, which was confirmed 
in all dimensions (Figures $\mathbf{4 d}$ and $\mathbf{4 e}$ ). The 3D reconstructions also demonstrated large regions of graft encapsulated in WGA+ glycoproteins, consistent with fibrosis ${ }^{36}$ (Figures $\mathbf{4 d}$ and $\mathbf{4 e}$ ). We have previously found fibrosis is resolved three months after engraftment into non-human primate hearts, but this physical barrier may hinder electrical integration with the host and contribute to the rare, transient arrhythmogenic potential of cardiac cell therapy grafts seen soon after cell transplantation ${ }^{35,37}$.

\section{Large field imaging and morphological assessment in human and mouse kidneys}

One apparent limitation of high-powered objectives with small fields of view and short working distances is that they could be unable to completely capture larger structures, such as human glomeruli. Using the same sample preparation technique, high resolution images were obtained in human and mouse normal adult kidneys showing clearly resolved vasculature, tubules, podocytes and nuclei. To image entire human glomeruli, which exceed $100 \mu \mathrm{m}$ in size, a $40 x$ oil objective $(N A=1.3)$ was used and displayed modest reduction in resolution (Figure $5 \mathbf{a}$ and Video 6). Tiling (i.e. stitching) multiple fields of view also demonstrated larger regions can be imaged without compromising resolution (Figure 5b and Videos 7-9).

\section{Volumetric quantification of cells and subcellular structures in intact tissues}

To demonstrate broader utility, we subjected liver sections from adult mice to analysis by this method (Figure 6 and Videos 9-11). The resulting images were used for volumetric quantifications, such as hepatocyte volume, nuclei volume, and quantifying the number of nuclei per cell (Figure 6). For instance, we found that $72.9 \%$ of adult mouse hepatocytes were binucleated. This percentage closely mirrors previous in vitro studies in isolated hepatocytes ${ }^{38}$ but is substantially higher than other reports from in situ analysis that reported $27.5 \%$ binucleation ${ }^{39}$, which likely underrepresented the amount of binucleation due to the use of $3 \mu \mathrm{m}$ thick sections that fail to capture whole hepatocytes. Binucleated hepatocyte volume was $64 \%$ larger compared to mononucleated hepatocytes (3614 $\pm 82.45 \mu \mathrm{m}^{3}$ versus $2202 \pm 84.63 \mu \mathrm{m}^{3}, \mathrm{P}<0.0001$, Figure 6), with the volume of individual nuclei showing no significant difference between these groups (159.6 $\pm 6.13 \mu \mathrm{m}^{3}$ in mononucleated hepatocytes versus $150.3 \pm 2.33 \mu^{3}$ in binucleated hepatocytes, $\mathrm{P}<0.14)$. Consistently, the proportion of nuclear volume to cytoplasm volume was similar between the groups, with the fraction of total nuclear volume being $19 \%$ higher in binucleated hepatocytes. $(0.081 \pm 0.0028$ in mononucleated hepatocytes versus $0.097 \pm 0.0022$ in binucleated hepatocytes, $\mathrm{P}<0.004$, Figure 6). 


\section{Discussion}

Here, we developed a combined histology and tissue clearing approach that is broadly applicable to studies of molecular and cellular three-dimensional organization in adult human and non-human mammalian specimens. Using common lab equipment and reagents, we show this method can be applied to studies of cardiac myocyte proliferation in adult human hearts samples; subnuclear chromatin architecture; cardiac myocyte gap junctions; human pluripotent stem cellderived cardiac cell therapy engraftment in host rats; human kidney tubules, glomeruli, and vasculature; liver vessel networks and hepatocyte canalicular networks; and volumetric quantification of cells, subcellular structures, and nuclei number in large cells in situ. Using this technique, we determined that adult $\mathrm{CMs}$ are not proliferative in healthy or diseased human hearts. High resolution 3D imaging of human pluripotent stem cell (PSC)-derived cardiac myocytes engrafted in host rats demonstrated grafts integrate with the host myocardium but also revealed large portions of graft are encapsulated by fibrosis 6 weeks after implantation. Imaging of liver samples with intact hepatocytes demonstrated unprecedented levels of binucleation that correlated with cell hypertrophy. Interestingly, these images also revealed spiral-aligned nuclei in the liver bile duct epithelium and kidney tubules. We confirmed this method can be used with multiple imaging modalities including conventional single-photon line-scanning confocal and spinning disk microscopy. This methodology could be adapted for 3D pathological assessments of disease state and for generating submicron-resolution whole organ atlases. Indeed, subcellular features are emerging as key markers for disease progression, such as multinucleation, sarcomere and T-tubule density and alignment, capillary density and alignment, and canaliculi dilation $^{27,40-47}$. In addition, recent tissue engineering approaches demonstrated cells and extracellular matrix can be bioprinted with micron-level resolution ${ }^{48,49}$, the datasets generated by this method will also be useful blueprints for engineering human tissues.

This method overcomes the limitations that have prevented high resolution 3D imaging of fluorescently labeled human biopsies, though, intrinsic limitations remain. For example, single photon confocal imaging illuminates the entire depth of the sample while imaging each plane, thus the sequential z-stack imaging could lead to photobleaching. Despite this we show this is not a limiting factor for most of the common fluorophores which have been designed with high extinction coefficients (i.e. Alexa Fluor conjugates and common DNA dyes) ${ }^{50}$. This issue could further be mitigated by two-photon imaging ${ }^{51}$. While this protocol maximizes the amount of specimen imageable with high powered objectives (i.e. $60 x$ oil, $N A=1.4$ ), which are required for submicron resolution in 3D, going beyond $100 \mu \mathrm{m}$ in depth is still hampered by the working distances of these objectives $(210 \mu \mathrm{m})$, which is partially occupied by the coverslip, with even the thinnest 
coverslips (i.e. \#0) being approximately $100 \mu \mathrm{m}$ thick. This can be overcome partially by using lower NA objectives (i.e. $40 x$ oil, $N A=1.3$ ) that have greater working distance, with modest reduction in resolution, enabling imaging of $>160 \mu \mathrm{m}$ of sample depth. This protocol quenches native fluorescence of genetically encoded fluorescent proteins. We overcame this by utilizing antibodies specific to green fluorescent protein (GFP), and such antibodies have been generated for all commonly used fluorescent proteins ${ }^{52}$. Lastly, the hundreds of z-stack images per specimen required for full utilization of the protocol can take over an hour to acquire and can be cumbersome to handle in image analysis software like ImageJ. We demonstrate these limitations can be ameliorated with faster imaging modalities (i.e. spinning disk confocal) and software (ie BitPlane Imaris, Nikon Elements) that enable faster acquisitions and analysis. Resonant scanning or light-sheet imaging are alternatives that could also increase acquisition speed ${ }^{53}$. Altogether this simple and rapid method achieves the highest levels of resolution and imaging depth that is possible using widely available standard confocal microscopy and broadly enables molecular biology and volumetric quantification studies in the context of three-dimensional human and mammalian tissues. 


\section{Materials and Methods}

The entire method is summarized in Figure 1a and the reagents and equipment used are listed in Table 1. Troubleshooting suggestions are provided in Table 2.

\section{Human sample preparation:}

All human samples were approved by the Institutional Review boards of the University of Washington in Seattle. Fresh samples were collected in cold $\left(4^{\circ} \mathrm{C}\right)$ PBS, or for heart tissue samples were collected in a high potassium buffer, KB buffer $\left(110 \mathrm{mM}\left[\mathrm{K}^{+}\right]\right)$, to keep the myocytes arrested. Tissues were trimmed tissue into $\sim 6 \mathrm{~mm}^{3}$ cubes or smaller. Samples were then transferred to a $50 \mathrm{~mL}$ conical tube with $30 \mathrm{~mL}$ of fresh $4 \%$ paraformaldehyde (PFA) in PBS, precooled to $4^{\circ} \mathrm{C}$ and incubated 3-6 hours at $4^{\circ} \mathrm{C}$ in a rotator. The samples were then transferred into a new $50 \mathrm{~mL}$ conical tube containing $30 \mathrm{~mL} 100 \%$ methanol $(\mathrm{MeOH})$, precooled to $-20^{\circ} \mathrm{C}$ and incubated at $-20^{\circ} \mathrm{C}$ for 1 hour. In a stepwise manner, samples were incubated in $30 \mathrm{~mL}$ of precooled $80 \% \mathrm{MeOH} / 20 \% \mathrm{PBS}, 60 \% \mathrm{MeOH} / 40 \% \mathrm{PBS}$, with each incubation for 30 minutes at $20^{\circ} \mathrm{C}$. The samples were then washed $3 x$ with precooled PBS and kept in PBS at $4^{\circ} \mathrm{C}$ until embedding.

\section{Rodent sample preparation:}

All animal protocols in this study were approved by the University of Washington Office of Animal Welfare (IACUC protocol \# 4290-01) and conform to the Guide for the Care and Use of Laboratory Animals published by the US National Institutes of Health. All mice studies were performed in animals 8-12 weeks of age. Cardiac cell therapy injections into immunocompromised adult rats were performed as described ${ }^{54}$. We administered 10 units of heparin per gram of body weight by intraperitoneal injection, allowed it to circulate for 10 minutes to prevent blood coagulation, and then euthanized the animals via isoflurane overdose. Whole organs were dissected from animals and collect into iced Petri dishes containing $4^{\circ} \mathrm{C}$ PBS or KB buffer (hearts). Extraneous tissue was trimmed. We cannulated aorta (hearts), portal vein (livers), or renal artery (kidneys) with a blunt $26 \mathrm{~g}$ needle, and secured the canula with a clip and suture for organ perfusion: We perfused $10 \mathrm{~mL}$ of PBS or KB buffer (hearts), precooled to $4^{\circ} \mathrm{C}$, with a syringe at a flow rate of $\sim 2 \mathrm{~mL} / \mathrm{min}$ to wash out blood cells. The syringe was then replaced with a new one containing $10 \mathrm{~mL} 4 \%$ PFA in PBS, precooled at $4^{\circ} \mathrm{C}$, and tissues were perfusion fixed at a flow rate of $\sim 2 \mathrm{~mL} / \mathrm{min}$. From here we followed the preparation as described for human samples, starting from incubation with $4 \%$ PFA for 3-6 hours at $4^{\circ} \mathrm{C}$ in a rotator through methanol fixation and PBS washes. 


\section{Tissue Embedding and Sectioning:}

$6 \%$ low-melt agarose in PBS was prepared in a $50 \mathrm{~mL}$ tube and dissolved by boiling the tube in a beaker containing water on a hotplate with the tube lid loose. The solution was cooled to $45^{\circ} \mathrm{C}$ in a water bath incubator. Fixed specimens were dabbed on a Kimwipe or gauze and placed in a cryomold that was then quickly filled with the molten agarose. Samples were left at room temperature to allow gelation and stored at $4^{\circ} \mathrm{C}$ until sectioning.

The agarose-tissue block was removed from the mold and the agarose was trimmed such that there was $\sim 4 \mathrm{~mm}$ of agarose surrounding each side of the tissue, keeping the sample on ice. The trimmed agarose block was superglued to a vibratome sectioning platform. The vibratome sample chamber was then filled with PBS, precooled to $4^{\circ} \mathrm{C}$. For sectioning, we found slow blade advancement and moderate amplitude to be crucial for good sectioning. For a Leica $1200 \mathrm{~S}$ vibratome we used $0.14 \mathrm{~mm} / \mathrm{min}$ blade advance speed, $0.5 \mathrm{~mm}$ amplitude, and a $15^{\circ}$ cut angle. Section thickness was set to $100-200 \mu \mathrm{m}$. We ensured the vibratome section thickness was calibrated using Leica Vibro-Check. Sections were collected with a soft, synthetic-bristle paintbrush and transferred to a 24-well plate containing cold PBS. Sections were stored up to two weeks at $4^{\circ} \mathrm{C}$ in PBS $+0.1 \%$ Na-azide $+1 \%$ bovine serum albumin (BSA), or for longer periods (we tested up to one year) at $-20^{\circ} \mathrm{C}$ in $10 \%$ DMSO $90 \%$ PBS with $2 \%$ BSA.

\section{In-suspension staining:}

We utilized a convenient staining protocol we developed specifically for vibratome sections. In contrast to other sections, vibratome sections remain free-floating in suspension throughout the staining protocol to improve reagent penetration. We used 24-well plates filled with PBS or staining reagents and home-made baskets to hold the sections and minimize handling. The sample baskets are plastic cylinders that have a nylon mesh at one end, allowing the exchange of fluids from the sample within the basket and the buffers in the 24-well plate wells. To make these, we trimmed the cap and bottom from a $5 \mathrm{~mL}$ polypropylene tube to make an open cylinder. We used nylon mesh (ie Cell Strainer with $100 \mu \mathrm{m}$ mesh) that was then placed a piece of aluminum foil on a hot plate and briefly pressed the plastic cylinder against the nylon mesh on top of the foil/hot plate to melt the mesh onto the tube edge. Other vessels may also be suitable, but it is important to stain the samples in suspension where reagents can flow through and make contact with all sides of the sample (in contrast to after being adhered to glass allowing only partial contact with reagents), as this improves the penetration of antibodies and dyes into the interior of the section. We prepared 24-well plates with the solutions below in sequential order, using 600uL of solution per well, and simply transferred the sample basket sequentially from well to well. 
Sections were transferred into sample baskets using a soft synthetic bristle paintbrush. Samples were washed with PBS $+0.1 \%$ TritonX100 (PBST) three times at room temperature. Blocking was performed with 5\% normal donkey serum in PBST for 1 hour at room temperature on an orbital rocker. Plates were then transferred to $4^{\circ} \mathrm{C}$ and rocked on orbital shaker for an additional 30 minutes. Samples were incubated in primary antibodies diluted in blocking buffer supplemented with $1 \% \mathrm{DMSO}$ at $4^{\circ} \mathrm{C}$ for 16 hours with rocking on orbital rocker. Samples were washed PBST four times, 5 minutes per wash, on orbital rocker at room temperature. In the last wash, plates were moved to $4^{\circ} \mathrm{C}$ on an orbital rocker for an additional 30 minutes. Samples were incubated with secondary antibodies + chemical stains (such as DAPI, Hoechst, phalloidin, or wheat germ agglutinin, WGA) at $4^{\circ} \mathrm{C}$ for 16 hours with rocking. Samples were then washed with PBST four times, 5 minutes per wash, on an orbital rocker at room temperature. Lastly, samples were kept in PBS until mounting.

\section{Clearing and Mounting:}

We prepared glass slides by adhering two pieces of $\sim 300 \mu \mathrm{m}$-thick solvent-resistant aluminum foil tape such that they were spaced far enough apart to accommodate the sample fitting in between them, but close enough so that both sides of the coverslip could rest on the foil. These acted as bridges for the coverslips to lean on, preventing the samples from being crushed or squeezed. 22x22 mm coverslips were coated with $0.01 \%$ poly-L-lysine in PBS. Sections were transferred from the basket into a Petri dish containing PBS and then onto the center of the coverslip with a paint brush. Residual PBS was blotted off. The coverslips with samples were placed in a coverslip rack/caddy, and submerged into glass jars containing $100 \mathrm{~mL}$ of following solutions sequentially, 50 seconds each incubation: 70\% isopropanol, $85 \%$ isopropanol, $95 \%$ isopropanol, $100 \%$ isopropanol x 2, Benzyl alcohol and benzyl benzoate at a 1:2 ratio (BABB) x3. The samples were kept in the final BABB incubation for at least 10 minutes and then transferred to the glass slide, placing the coverslip between the two foil bridges. Excess BABB was blotted off and the slides were sealed with 3 coats of nail polish

\section{Imaging:}

All images were obtained using standard methods on a Nikon A1R confocal microscope, except for Figure 2e where a Nikon TiE equipped with Yokogawa spinning disk head was used and Figure 4a-b where a widefield Nikon TiE microscope was used. A 60x oil NA=1.4 objective was used for all images, except for Figure 4a-b where a 10x dry objective was used and Figure 5 a where a $40 x$ oil $N A=1.3$ objective was used. Unless noted otherwise in the figure legend, all $z-$ 
stacks were acquired with a $60 x$ oil objective and $300 \mathrm{~nm}$ z-step size, resulting in a voxel size of $<0.012 \mu \mathrm{m}^{3}$. While our microscopy configurations did not require adjustments when using \#0 coverslips, coverslip thickness or the correction collar on objective lenses could be adjusted to circumvent imaging aberrations if seen with the fluorescent bead standards ${ }^{55}$.

\section{Visualization and analysis:}

ImageJ/FIJI was used to generate orthogonal slices of the 3D reconstructed z-stacks (Menu: Images $\rightarrow$ Stacks $\rightarrow$ Orthogonal views). Z-intensity profiling was performed for each channel using Image $J$ (Menu: Images $\rightarrow$ Stacks $\rightarrow$ Plot Z-axis profile). We used ImageJ, Nikon Elements, and BitPlane Imaris to generate 3D renderings. Imaris was used for generating videos of orthogonal slices through the tissue volumes. For analysis of hepatocyte volume and nuclei we used Imaris V9.2 and the Imaris Surfaces and Cell modules. Nuclei (Hoechst), hepatocytes (Factin), and liver vasculature (WGA) were segmented independently. The segmented vasculature mask was applied to the nuclei channel and subtracted to remove non-hepatocyte nuclei. The remaining nuclei were masked and the number of nuclear masks per segmented hepatocyte was quantified. 


\section{Acknowledgements}

We would like to acknowledge April Stempien-Otero and Chris P. Miller for providing human samples. We are grateful to Dale Hailey and the University of Washington's Garvey Imaging Core for microscopy support. We thank William C. Smith and Michael T. Veeman for the development of whole-mount embryo clearing protocols and consultation. This work was supported by AHA 14PRE20140025 and NIH F32HL143851 (DE), NIH R01 HL70748 (WRM), and by NIH grants R01HL128362, U54DK107979, R01HL128368, R01HL141570, R01HL146868, and grants from the Foundation Leducq Transatlantic Network of Excellence and Robert B. McMillen Foundation (CEM).

\section{Contributions}

D.E. designed the study, performed experiments, analyzed results, and wrote the manuscript. A.M.M. performed cardiac cell therapy surgeries and animal care. X.Y. generated PSC-derived cardiac myocytes for cell therapy. W.R.M., C.E.M., and X.Y. edited the manuscript. W.R.M. and C.E.M. provided supervision and acquired funding.

\section{Competing Interests}

W.R.M. and C.E.M. are scientific founders and equity holders in Cytocardia. The other authors declare no competing interests. 


\section{References}

1. Titford, M. A Short History of Histopathology Technique. J. Histotechnol. 29, 99-110 (2006).

2. Richardson, D. S. \& Lichtman, J. W. Clarifying Tissue Clearing. Cell 162, 246-257 (2015).

3. Eisenstein, M. Transparent tissues bring cells into focus for microscopy. Nature 564, 147-149 (2018).

4. Jing, D. et al. Tissue clearing of both hard and soft tissue organs with the PEGASOS method. Cell Res. 28, 803-818 (2018).

5. Chung, K. et al. Structural and molecular interrogation of intact biological systems. Nature 497, 332-337 (2013).

6. Glaser, A. K. et al. Multi-immersion open-top light-sheet microscope for high-throughput imaging of cleared tissues. Nat. Commun. 10, 2781 (2019).

7. Tomer, R., Ye, L., Hsueh, B. \& Deisseroth, K. Advanced CLARITY for rapid and highresolution imaging of intact tissues. Nat. Protoc. 9, 1682-1697 (2014).

8. Murakami, T. C. et al. A three-dimensional single-cell-resolution whole-brain atlas using CUBIC-X expansion microscopy and tissue clearing. Nat. Neurosci. 21, 625-637 (2018).

9. Blanca, C. \& Hell, S. Axial superresolution with ultrahigh aperture lenses. Opt. Express 10, 893 (2002).

10. Ke, M.-T., Fujimoto, S. \& Imai, T. SeeDB: a simple and morphology-preserving optical clearing agent for neuronal circuit reconstruction. Nat. Neurosci. 16, 1154-1161 (2013).

11. Engerer, P., Fecher, C. \& Misgeld, T. Super-resolution microscopy writ large. Nat. Biotechnol. 34, 928-930 (2016).

12. Renier, N. et al. iDISCO: A Simple, Rapid Method to Immunolabel Large Tissue Samples for Volume Imaging. Cell 159, 896-910 (2014).

13. Kohl, J. et al. Ultrafast tissue staining with chemical tags. doi:10.1073/pnas.1411087111

14. Hama, H. et al. Scale: a chemical approach for fluorescence imaging and reconstruction of transparent mouse brain. Nat. Neurosci. 14, 1481-1488 (2011).

15. Foster, D. S. et al. A Clearing Technique to Enhance Endogenous Fluorophores in Skin and Soft Tissue. Sci. Rep. 9, (2019).

16. Benninger, R. K. P. \& Piston, D. W. Two-photon excitation microscopy for the study of living cells and tissues. Curr. Protoc. cell Biol. Chapter 4, Unit 4.11.1-24 (2013).

17. Trägårdh, J. et al. Exploration of the two-photon excitation spectrum of fluorescent dyes at wavelengths below the range of the Ti:Sapphire laser. J. Microsc. 259, 210-8 (2015). 
18. Drobizhev, M., Makarov, N. S., Tillo, S. E., Hughes, T. E. \& Rebane, A. Two-photon absorption properties of fluorescent proteins. Nat. Methods 8, 393-9 (2011).

19. Liu, Y.-C. \& Chiang, A.-S. High-resolution confocal imaging and three-dimensional rendering. Methods 30, 86-93 (2003).

20. Dekkers, J. F. et al. High-resolution 3D imaging of fixed and cleared organoids. Nat. Protoc. 14, 1756-1771 (2019).

21. Lin, A. Y. et al. Rigid Embedding of Fixed and Stained, Whole, Millimeter-Scale Specimens for Section-free 3D Histology by Micro-Computed Tomography. J. Vis. Exp. e58293 (2018). doi:10.3791/58293

22. Khimchenko, A. et al. Extending two-dimensional histology into the third dimension through conventional micro computed tomography. Neuroimage 139, 26-36 (2016).

23. El-Nachef, D. et al. Repressive histone methylation regulates cardiac myocyte cell cycle exit. J. Mol. Cell. Cardiol. 121, 1-12 (2018).

24. van Berlo, J. H. \& Molkentin, J. D. An emerging consensus on cardiac regeneration. Nat. Med. 20, 1386-1393 (2014).

25. Oyama, K., El-Nachef, D. \& Maclellan, W. R. Regeneration potential of adult cardiac myocytes. Cell Res. 23, (2013).

26. Oyama, K., El-Nachef, D., Zhang, Y., Sdek, P. \& MacLellan, W. R. Epigenetic regulation of cardiac myocyte differentiation. Front. Genet. 5, (2014).

27. Laflamme, M. A. \& Murry, C. E. Heart regeneration. Nature 473, 326-335 (2011).

28. Tresser, J. et al. doublesex/mab3 related-1 (dmrt1) is essential for development of anterior neural plate derivatives in Ciona. Development 137, (2010).

29. Veeman, M. T., Newman-Smith, E., El-Nachef, D. \& Smith, W. C. The ascidian mouth opening is derived from the anterior neuropore: Reassessing the mouth/neural tube relationship in chordate evolution. Dev. Biol. 344, (2010).

30. Xiao, G. et al. Inducible activation of c-Myc in adult myocardium in vivo provokes cardiac myocyte hypertrophy and reactivation of DNA synthesis. Circ. Res. 89, 1122-9 (2001).

31. Karbassi, E. et al. Direct visualization of cardiac transcription factories reveals regulatory principles of nuclear architecture during pathological remodeling. J. Mol. Cell. Cardiol. 128, 198-211 (2019).

32. Cowan, J., Li, D., Gonzalez-Quintana, J., Morales, A. \& Hershberger, R. E. Morphological analysis of 13 LMNA variants identified in a cohort of 324 unrelated patients with idiopathic or familial dilated cardiomyopathy. Circ. Cardiovasc. Genet. 3, 6-14 (2010).

33. Movassagh, M. et al. Distinct epigenomic features in end-stage failing human hearts. 
Circulation 124, 2411-22 (2011).

34. Laflamme, M. A. et al. Cardiomyocytes derived from human embryonic stem cells in prosurvival factors enhance function of infarcted rat hearts. Nat. Biotechnol. 25, 1015-1024 (2007).

35. Chong, J. J. H. et al. Human embryonic-stem-cell-derived cardiomyocytes regenerate non-human primate hearts. Nature 510, 273-277 (2014).

36. Emde, B., Heinen, A., Gödecke, A. \& Bottermann, K. Wheat germ agglutinin staining as a suitable method for detection and quantification of fibrosis in cardiac tissue after myocardial infarction. Eur. J. Histochem. 58, 2448 (2014).

37. Liu, Y.-W. et al. Human embryonic stem cell-derived cardiomyocytes restore function in infarcted hearts of non-human primates. Nat. Biotechnol. 36, 597-605 (2018).

38. Klaunig, J. E. et al. Mouse liver cell culture. I. Hepatocyte isolation. In Vitro 17, 913-25 (1981).

39. Guidotti, J.-E. et al. Liver cell polyploidization: a pivotal role for binuclear hepatocytes. J. Biol. Chem. 278, 19095-101 (2003).

40. Rogers, A. B. \& Dintzis, R. Z. Liver and Gallbladder. in Comparative Anatomy and Histology 193-201 (Elsevier Inc., 2012). doi:10.1016/B978-0-12-381361-9.00013-5

41. Wang, C. et al. The extent of liver injury determines hepatocyte fate toward senescence or cancer article. Cell Death Dis. 9, (2018).

42. Sun, H. C. et al. Microvessel density of hepatocellular carcinoma: Its relationship with prognosis. J. Cancer Res. Clin. Oncol. 125, 419-426 (1999).

43. Murakami, K., Kasajima, A., Kawagishi, N., Ohuchi, N. \& Sasano, H. Microvessel density in hepatocellular carcinoma: Prognostic significance and review of the previous published work. Hepatol. Res. 45, 1185-1194 (2015).

44. Forster, J., Harriss-Phillips, W., Douglass, M. \& Bezak, E. A review of the development of tumor vasculature and its effects on the tumor microenvironment. Hypoxia Volume 5, 2132 (2017).

45. Gentric, G., Desdouets, C. \& Celton-Morizur, S. Hepatocytes Polyploidization and Cell Cycle Control in Liver Physiopathology. Int. J. Hepatol. 2012, 1-8 (2012).

46. Ibrahim, M., Gorelik, J., Yacoub, M. H. \& Terracciano, C. M. The structure and function of cardiac t-tubules in health and disease. Proceedings of the Royal Society B: Biological Sciences 278, 2714-2723 (2011).

47. Harvey, P. A. \& Leinwand, L. A. Cellular mechanisms of cardiomyopathy. Journal of Cell Biology 194, 355-365 (2011). 
48. Lee, A. et al. 3D bioprinting of collagen to rebuild components of the human heart. Science 365, 482-487 (2019).

49. Miri, A. K. et al. Effective bioprinting resolution in tissue model fabrication. Lab Chip 19, 2019-2037 (2019).

50. Sabnis, R. W. Handbook of Fluorescent Dyes and Probes. Handbook of Fluorescent Dyes and Probes (2015). doi:10.1002/9781119007104

51. Denk, W., Strickler, J. \& Webb, W. Two-photon laser scanning fluorescence microscopy. Science (80-. ). 248, 73-76 (1990).

52. Cai, D., Cohen, K. B., Luo, T., Lichtman, J. W. \& Sanes, J. R. Improved tools for the Brainbow toolbox. Nat. Methods 10, 540-547 (2013).

53. Greer, C. J. \& Holy, T. E. Fast objective coupled planar illumination microscopy. Nat. Commun. 10, (2019).

54. Bargehr, J. et al. Epicardial cells derived from human embryonic stem cells augment cardiomyocyte-driven heart regeneration. Nat. Biotechnol. 37, 895-906 (2019).

55. Dumitriu, D., Rodriguez, A. \& Morrison, J. H. High-throughput, detailed, cell-specific neuroanatomy of dendritic spines using microinjection and confocal microscopy. Nat. Protoc. 201169 6, 1391 (2011). 


\section{Figure Legends}

Figure 1 Overview of method and quality control. (a) Overview of protocol showing major steps and timing from tissue harvesting to imaging. Scale bar=4 $\mathrm{mm}$. (b) Quality control representative images of fluorescent microbeads of different sizes mounted in BABB with a \#0 coverslip, as done with subsequent tissue samples. The large image is a maximum intensity image of the z-stack with the boxed regions being 3.75, 6.26, and $12.73 \mu^{2}$ and enclosing 3,5 , or $10 \mu \mathrm{m}$ beads, respectively. Note the $10 \mu \mathrm{m}$ beads are only fluorescently labeled at the bead surface. The boxed regions were examined with orthogonal slices of the z-stack and the maximum diameter slice for each dimension are shown in representative images and quantified. (c) Quality control for signal intensity through $100 \mu \mathrm{m}$ of cardiac tissue using Z-intensity profiling. The average signal intensity per z-slice confirmed uniform staining and clearing for various fluorescent stains. (d) Representative 3D reconstruction of adult normal human heart. F-actin (red), WGA (green), and Hoechst (blue) staining from 373 confocal images, z-step size=300 nm. Acquired with $60 x$ oil $\mathrm{NA}=1.4$ objective.

Figure 2 Adult cardiac myocyte cell cycling. (a) Normal human, (b) human cardiomyopathy sample from left-ventricular assist device core biopsy, (c) wildtype mouse, and (d) cardiac myocyte specific Myc-activation transgenic mouse samples stained with F-actin (red), WGA (green), Hoechst (blue), and pH3 (white). Orthogonal slices, indicated by the yellow cross-lines, from 3D reconstructions are shown. (e) Spinning disk microscopy image of wildtype mouse sample. (a) 373 confocal images $z$-step size=300 nm, (b) 271 confocal images z-step size=300 $\mathrm{nm}$, (c) 102 confocal images $z$-step size=1 $\mu \mathrm{m}$, (d) 242 confocal images $z$-step size=300 nm, (e) 345 confocal images $z$-step size $=300 \mathrm{~nm}$. All images were acquired with $60 x$ oil NA=1.4 objective.

Figure 3 Adult cardiac myocyte heterochromatin and subnuclear chromatin domains. (a) Wildtype mouse cardiac sections were stained with WGA (green), Hoechst (blue), and heterochromatin markers histone $\mathrm{H} 3$ lysine 9 trimethylation (H3K9me3, red) and Heterochromatin Protein 1 gamma (HP1g, magenta). Insets, a single cardiac myocyte nucleus cropped from the zstack shows colocalization of H3K9me3 and HP1g at subnuclear heterochromatin domains. (b) H3K9me3 (white) staining in wildtype mouse cardiac sample. (c) Three cardiac myocyte nuclei were cropped showing heterogeneous nuclear morphology. Each image represents a $90^{\circ}$ rotation around the z-axis. (a-b) 270 confocal images $z$-step size $=300 \mathrm{~nm}$. All images were acquired with $60 x$ oil $N A=1.4$ objective. 
Figure 4 Cardiac cell therapy engraftment and myocyte gap junctions. Low magnification widefield images of immune compromised (athymic, cyclosporin treated) adult rat cardiac sections 6-weeks after injection with (a) vehicle control or (b) GFP expressing human embryonic stem cells differentiated to cardiac myocytes (hESC-CM), stained with GFP (magenta) WGA (green) and Hoechst (blue). Inset shows F-actin staining of the same region of graft in an adjacent section, bar=10 $\mu \mathrm{m}$. (c) Vehicle injected rat cardiac section stained for GFP (magenta), Connexin 43 (Cx43, red), WGA (green) and Hoechst (blue) showing gap junctions. 165 confocal images z-step size=300 nm. Bar=20 $\mu \mathrm{m}$. (d) hESC-CM injected rat sections stained for GFP (magenta), Connexin 43 (Cx43, red), WGA (green) and Hoechst (blue) GFP+ graft and gap junctions demonstrating large patches of fibrosis around graft. 345 confocal images z-step size=300 nm. Bar $=20 \mu \mathrm{m}$. (e) Example of well-integrated graft showing each stain individually and a 3D reconstruction of the graft-host interface. 135 confocal images z-step size=300 nm. Bar=10 $\mu \mathrm{m}$. (c-e) Orthogonal slices, indicated by the yellow cross-lines, from 3D reconstructions are shown from images acquired with a $60 x$ oil $N A=1.4$ objective.

Figure 5 Adult kidney glomeruli and tubules. (a) Normal human orthogonal, left, and oblique slices, right, and (b) wildtype mouse orthogonal slices of kidney sections stained with F-actin (red) and Hoechst (green). (a) 534 confocal images $z$-step size $=300 \mathrm{~nm}$ acquired with a 40x oil NA=1.3 objective. (b) 253 confocal images, $z$-step size $=300 \mathrm{~nm}$ acquired with a $60 x$ oil NA=1.4 objective with $4 \times 4$ fields of view tiled to examine larger area. Left, tiled image, bar=200 $\mu \mathrm{m}$. Right, zoom in on boxed region in tiled image with orthogonal slices, bar $=10 \mu \mathrm{m}$.

Figure 6 Adult liver vasculature and quantification of hepatocyte nuclei number. (a) Oblique slices of 3D reconstruction showing portal vein, bile duct, hepatocytes, vasculature, and nuclei stained with F-actin (red), WGA (green), and Hoechst (blue). 395 confocal images z-step size $=300 \mathrm{~nm}$. Acquired with $60 x$ oil NA=1.4 objective. (b) Top left, segmentation of all nuclei. Middle left, nuclei colocalized with the vasculature. Bottom left, hepatocyte nuclei (nuclei from middle left were subtracted from top left), bars $=10 \mu \mathrm{m}$. Right, segmentation of mononucleated hepatocytes (red), binucleated hepatocytes (turquoise), and vasculature (green). (c) Quantification of on mononucleated (single) and binucleated (bi) hepatocyte volume. (d) Total nuclear to cytoplasmic ratio in mononucleated and binucleated hepatocytes. 


\section{Table legends}

Table 1 Equipment and Reagents. List of equipment and reagents used in this study. * indicates hazardous reagent or equipment, please consult user manuals and MSDS for appropriate usage.

Table 2 Suggested Troubleshooting. Potential problems and solutions when using this method.

\section{Video legends}

Video 1 Normal adult human heart. F-actin (red), WGA (green), and Hoechst (blue) staining 3D reconstruction from 373 confocal images, z-step size=300 nm. Acquired with $60 x$ oil NA=1.4 objective.

Video 2 Normal adult human heart. 3D reconstruction of the same images in Supplementary Video 1, with F-actin signal omitted to highlight T-tubules. Acquired with $60 x$ oil NA=1.4 objective.

Video 3 Wildtype adult mouse heart. F-actin (red), WGA (green), Hoechst (blue), and pH3 (white) staining 3D reconstruction from 102 confocal images, $z$-step size $=1 \mu \mathrm{m}$. Acquired with $60 \mathrm{x}$ oil NA=1.4 objective.

Video 4 Transgenic adult mouse heart with adult cardiac myocyte specific activation of the Myc oncogene. F-actin (red), WGA (green), Hoechst (blue), and pH3 (white) staining 3D reconstruction from 242 confocal images, z-step size $=300 \mathrm{~nm}$. Acquired with $60 \mathrm{x}$ oil NA=1.4 objective.

Video 5 Adult rat heart gap junction staining. Connexin 43 (red), WGA (green), and Hoechst (blue) staining 3D reconstruction from 165 confocal images, z-step size $=300 \mathrm{~nm}$. Acquired with $60 x$ oil $N A=1.4$ objective.

Video 6 Normal adult human kidney glomerulus. F-actin (red) and Hoechst (green) staining $3 D$ reconstruction from 534 confocal images, $z$-step size $=300 \mathrm{~nm}$. Acquired with $40 x$ oil NA=1.3 objective. 
Video 7 Normal adult human kidney tiled. F-actin (red) and Hoechst (green) staining 3D reconstruction from 253 confocal images, z-step size $=300 \mathrm{~nm}$. $4 \times 4$ fields of view were tiled to examine larger area. Acquired with $60 x$ oil $N A=1.4$ objective.

Video 8 Wildtype adult mouse kidney tiled. F-actin (red) and Hoechst (green) staining 3D reconstruction from 373 confocal images, $z$-step size $=300 \mathrm{~nm}$. $3 \times 3$ fields of view were tiled to examine larger area. Acquired with $60 x$ oil $N A=1.4$ objective.

Video 9 Wildtype adult mouse liver tiled. F-actin (white) staining 3D reconstruction from 435 confocal images, z-step size $=300 \mathrm{~nm}$. $3 \times 3$ fields of view were tiled to examine larger area. Acquired with $60 x$ oil $\mathrm{NA}=1.4$ objective.

Video 10 Wildtype adult mouse liver. F-actin (red), WGA (green), and Hoechst (blue) staining $3 D$ reconstruction from 395 confocal images, $z$-step size $=300 \mathrm{~nm}$. Acquired with $60 x$ oil NA=1.4 objective.

Video 11 Wildtype adult mouse liver vasculature. WGA (green) staining 3D reconstruction from 395 confocal images, $z$-step size $=300 \mathrm{~nm}$. Acquired with $60 x$ oil NA=1.4 objective. 
bioRxiv preprint doi: https://doi.org/10.1101/855254; this version posted November 26, 2019. The copyright holder for this preprint (which was

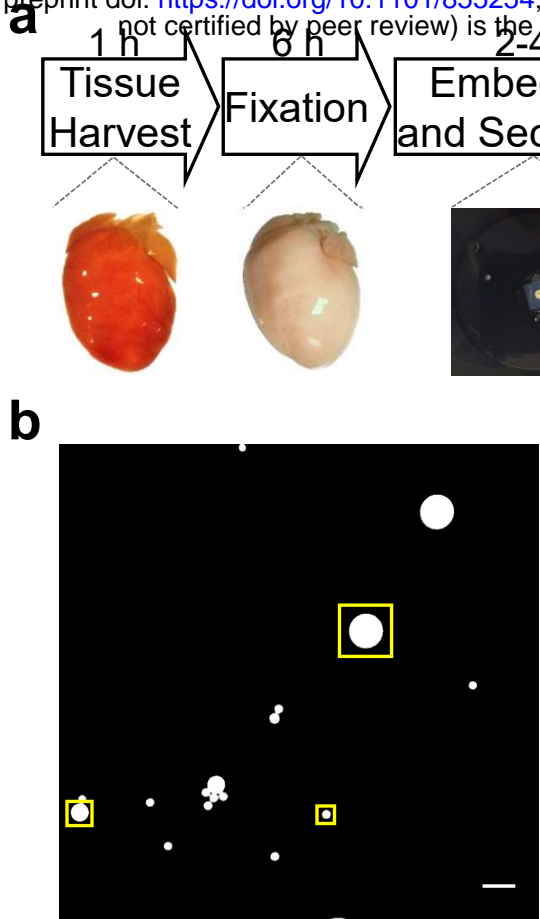

C

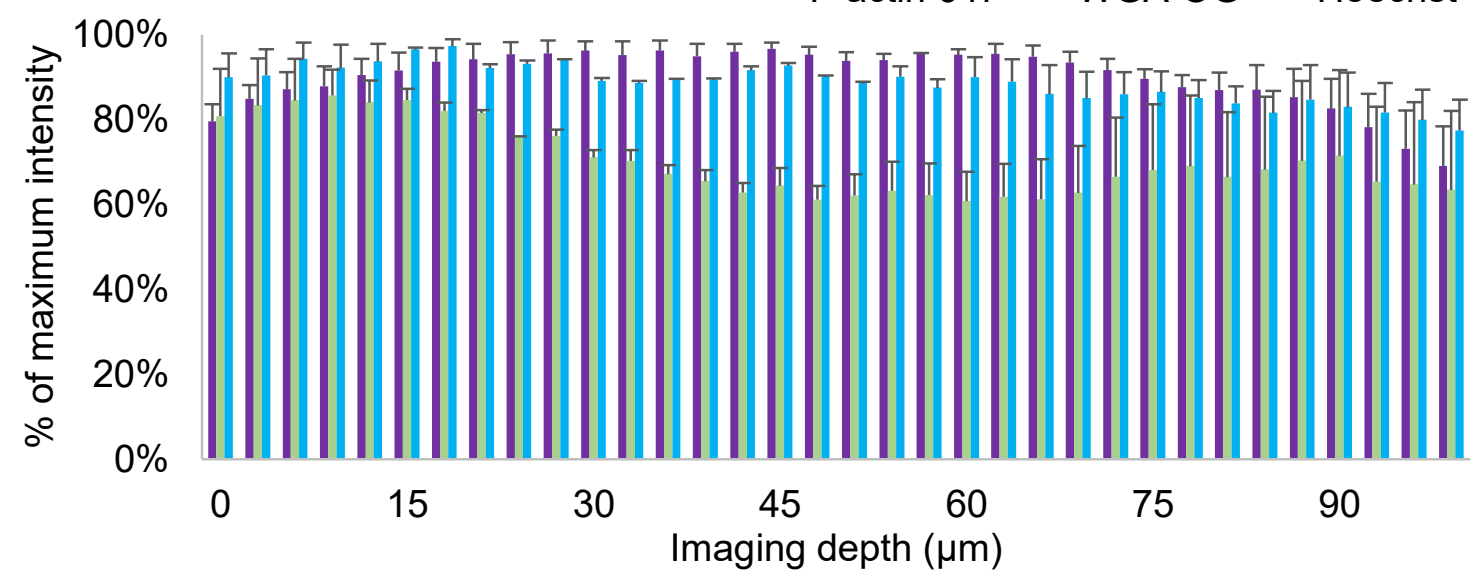

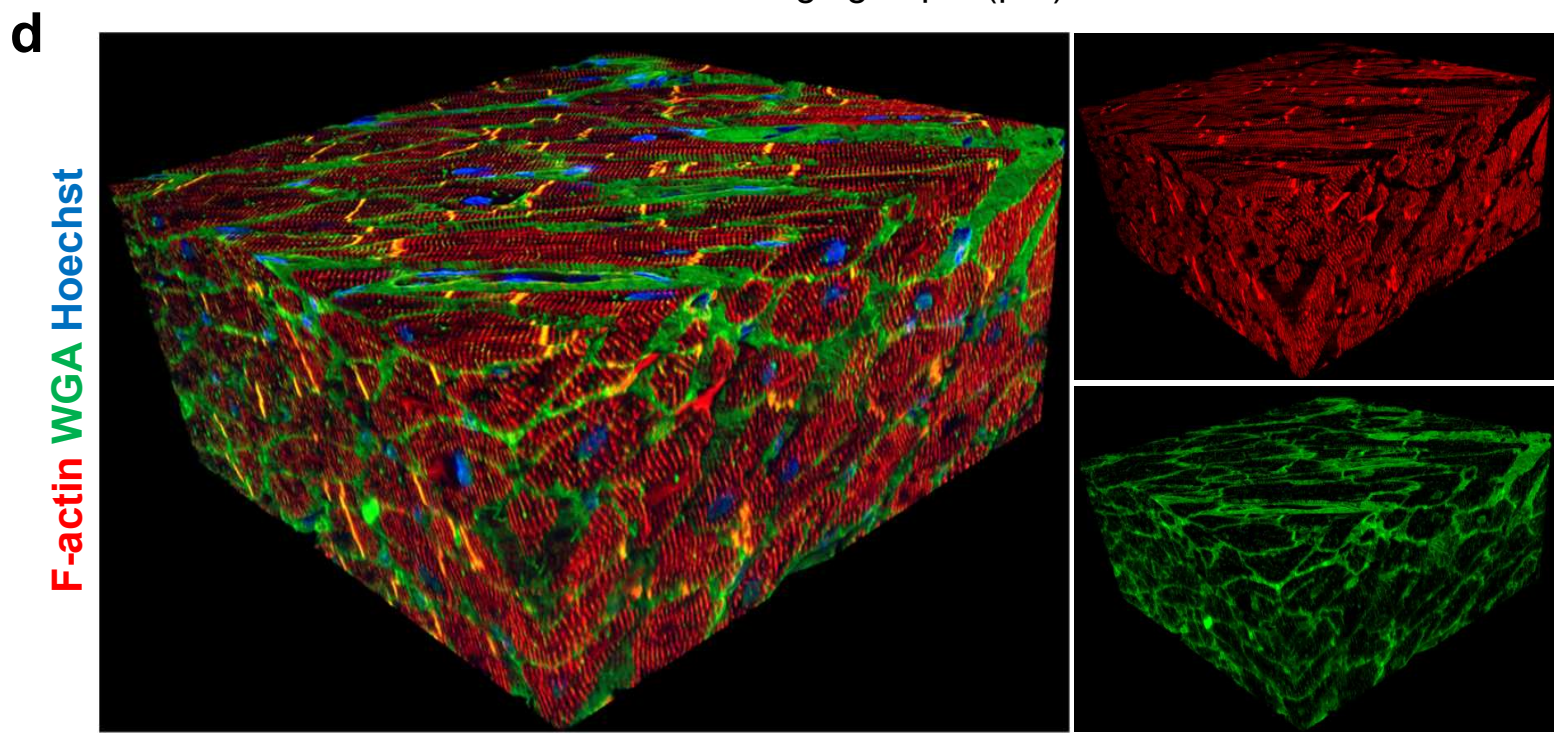

Figure 1 
bioRxiv preprint doi: https://doi.org/10.1101/855254; this version posted November 26, 2019. The copyright holder for this preprint (which was

a
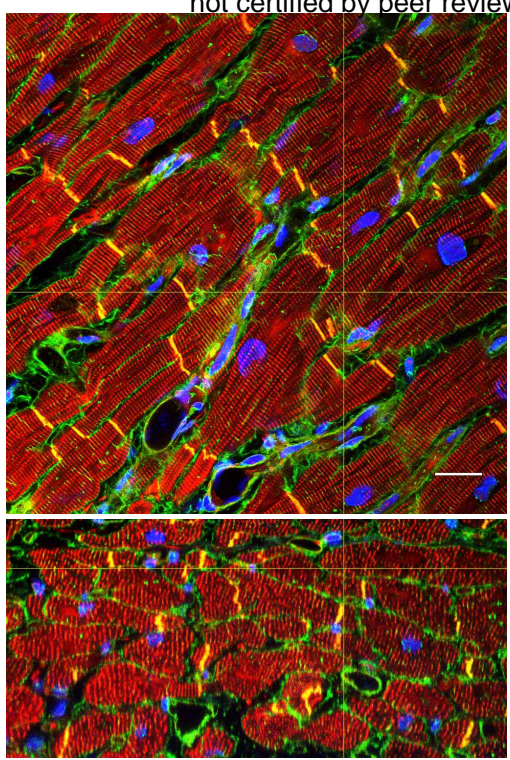

C
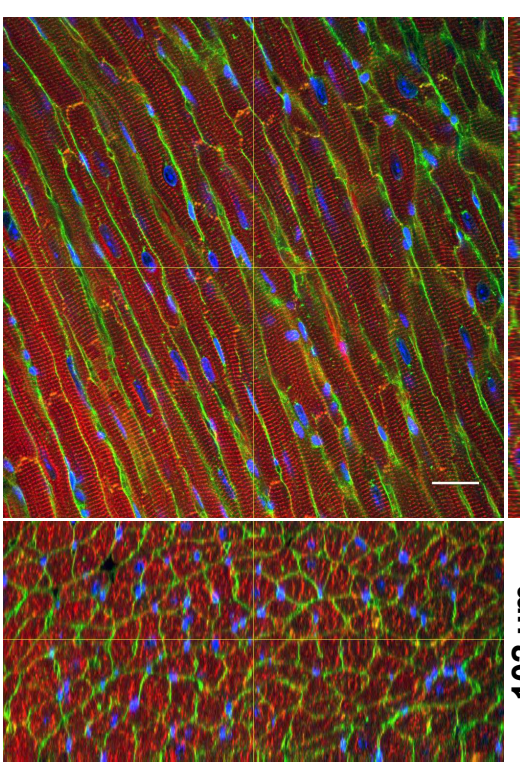

e

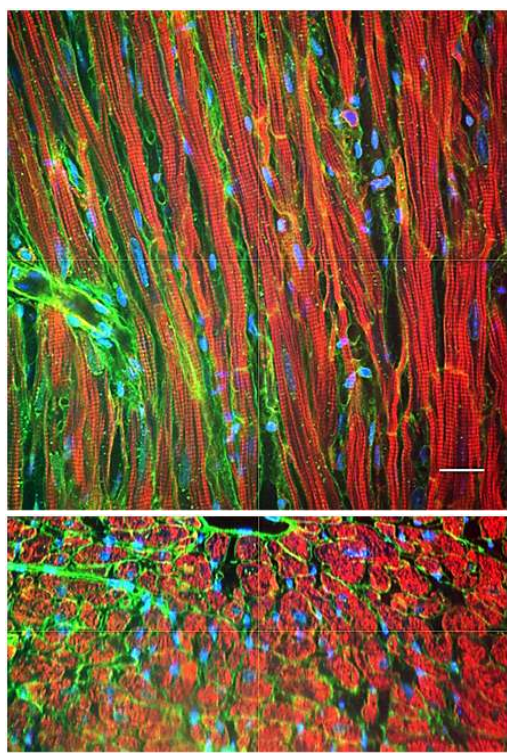

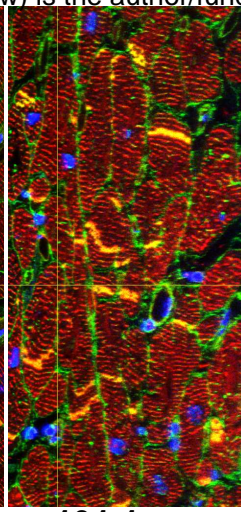

101.1 $\mu \mathrm{m}$

छ $\mathrm{pH} 3$

F-actin

WGA

은 Hoechst
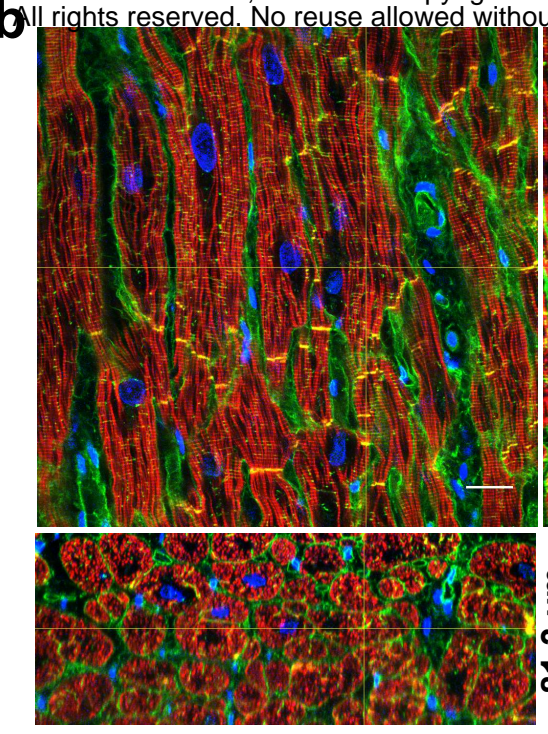

$81.3 \mu \mathrm{m}$

E $\mathrm{pH}_{3}$

F-actin

- WGA

$\infty$ Hoechst

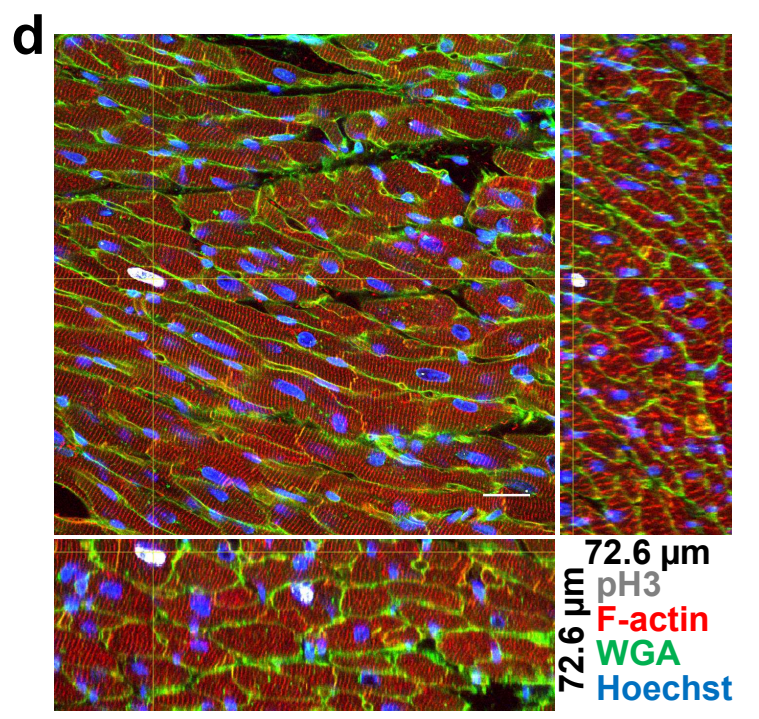

\section{E $\mathrm{pH} 3$}

F-actin

ㄴ WGA

Hoechst

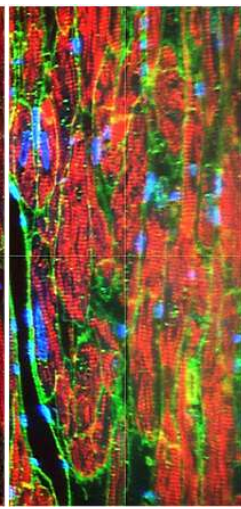

$103.5 \mu \mathrm{m}$

ह $\mathrm{pH} 3$

in F-actin

WGA

은 Hoechst

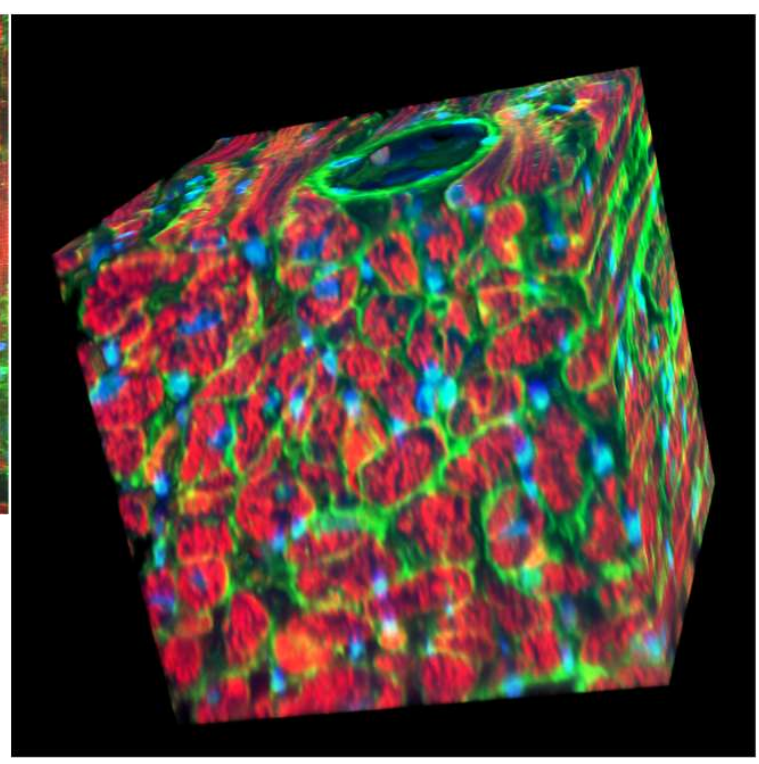

Figure 2 
bioRxiv preprint doi: https://doi.org/10.1101/855254; this version posted November 26,2019 . The copyright holder for this preprint (which was not certified by peer review) is the author/funder. All rights reserved. No reuse allowed without permission.

a

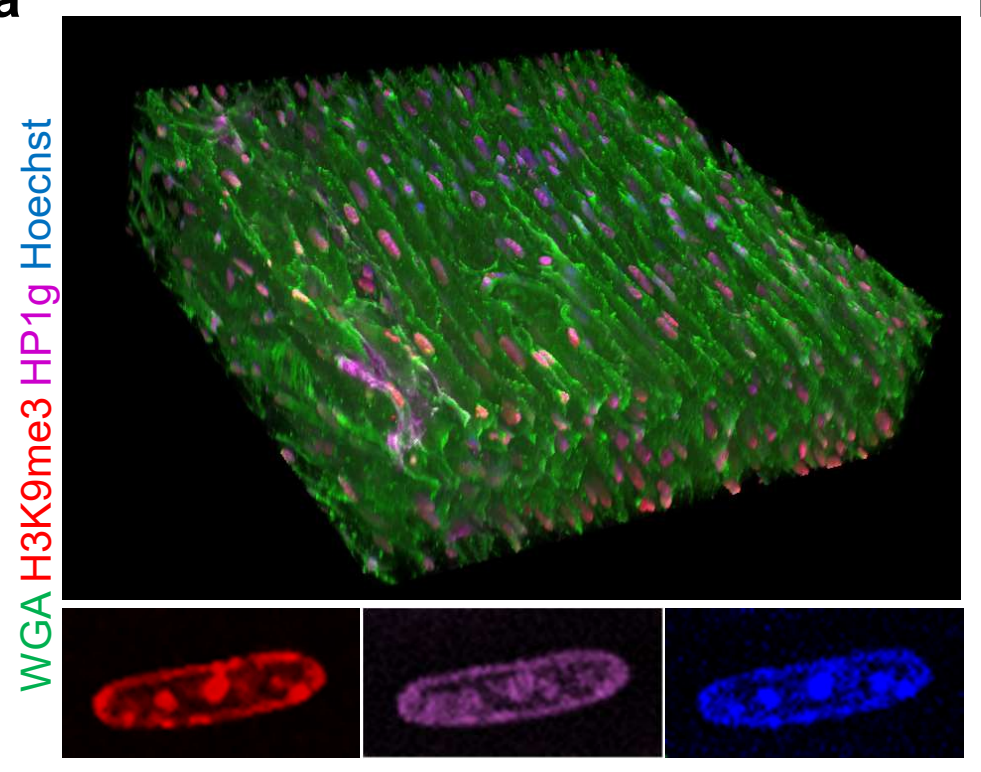

b

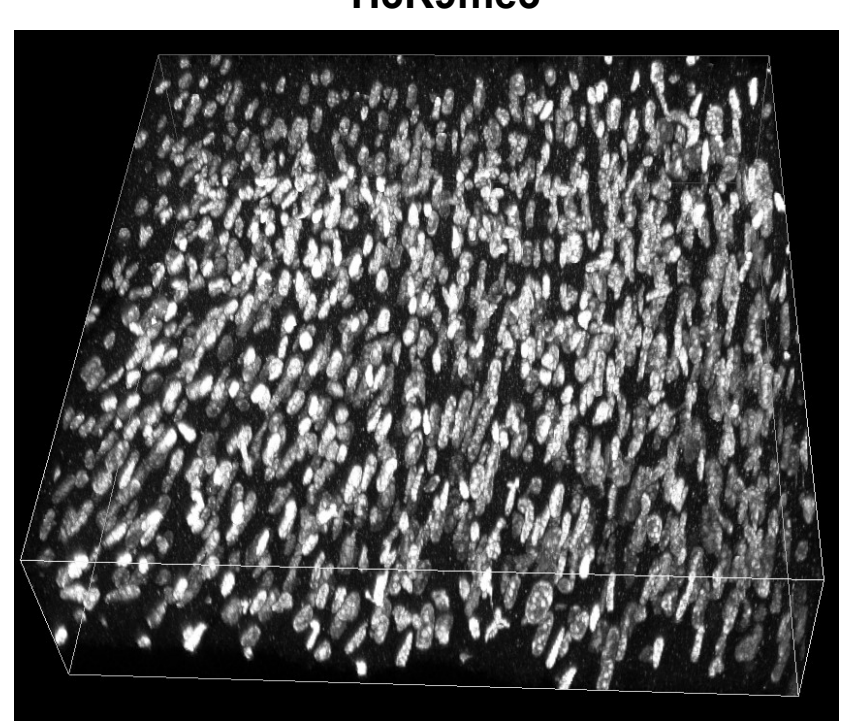

C
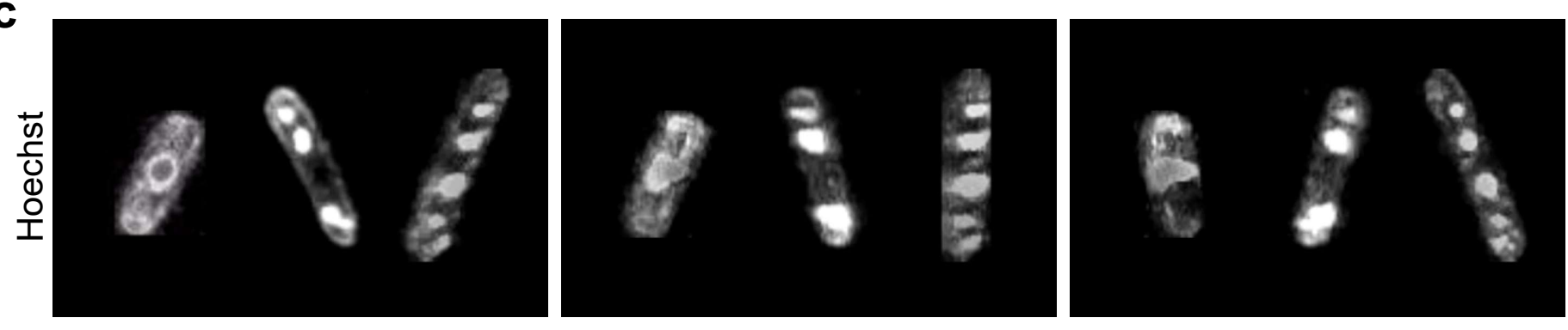

Figure 3 
bioRxiv preprint doi: https://doi.org/10.1101/855254; this version posted November 26,2019 . The copyright holder for this preprint (which was a not certified by peer review) is the author/funder. All rights reserved. No reuse allowed without permission.
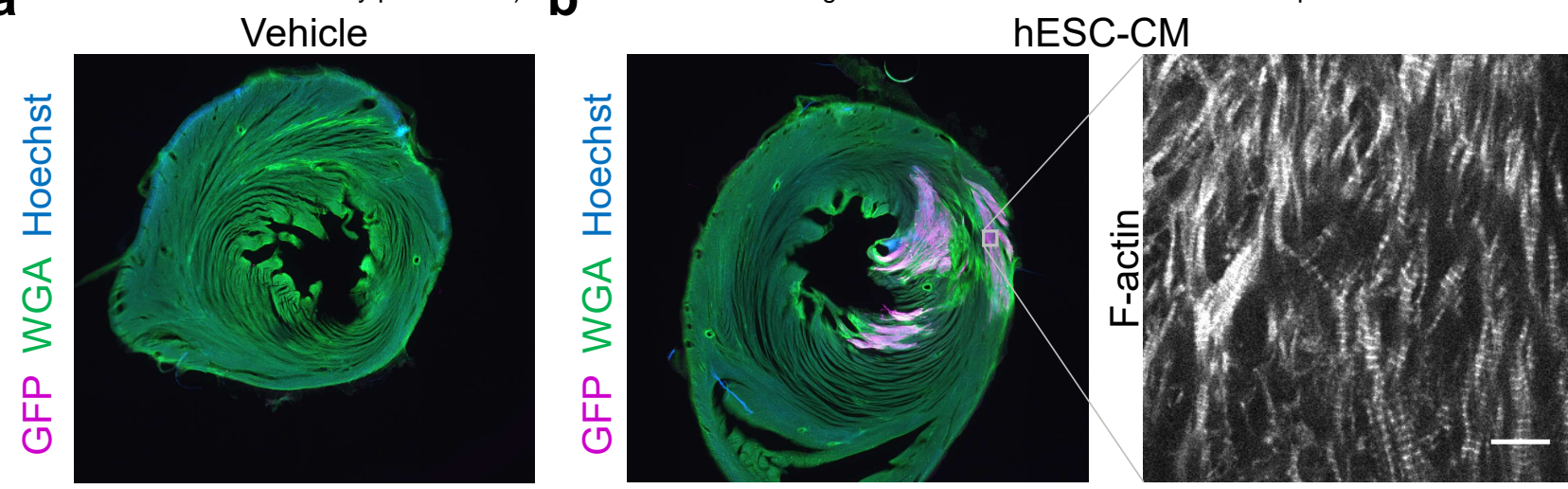

C

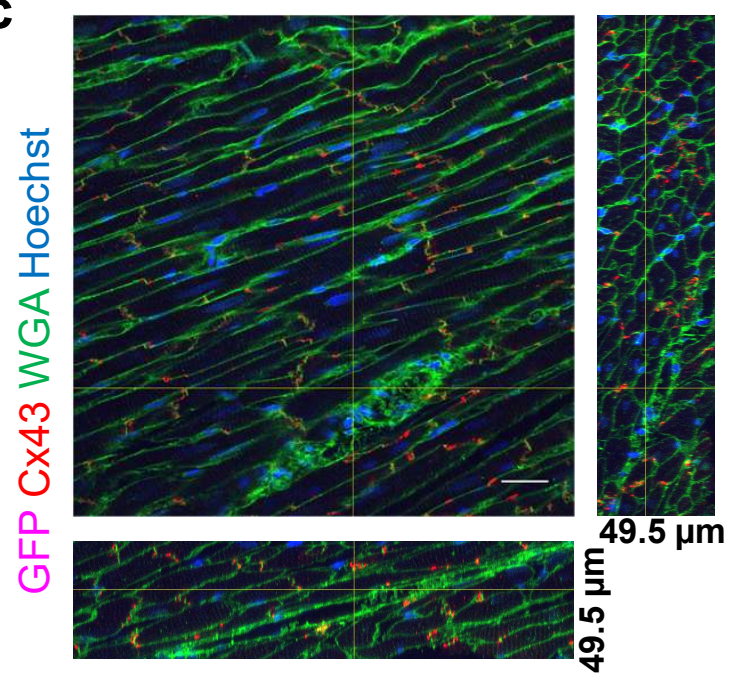

e

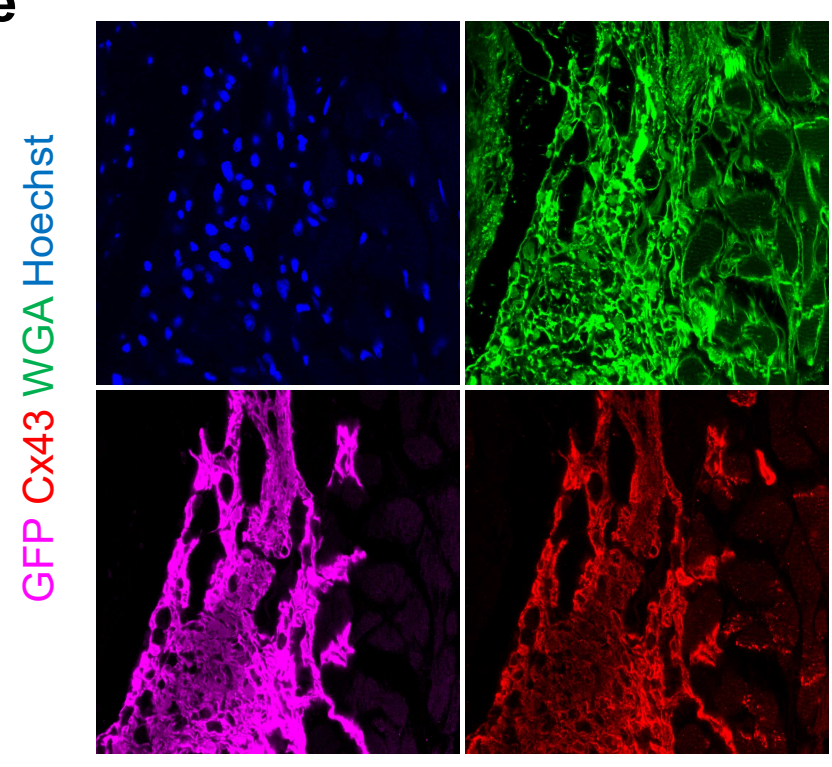

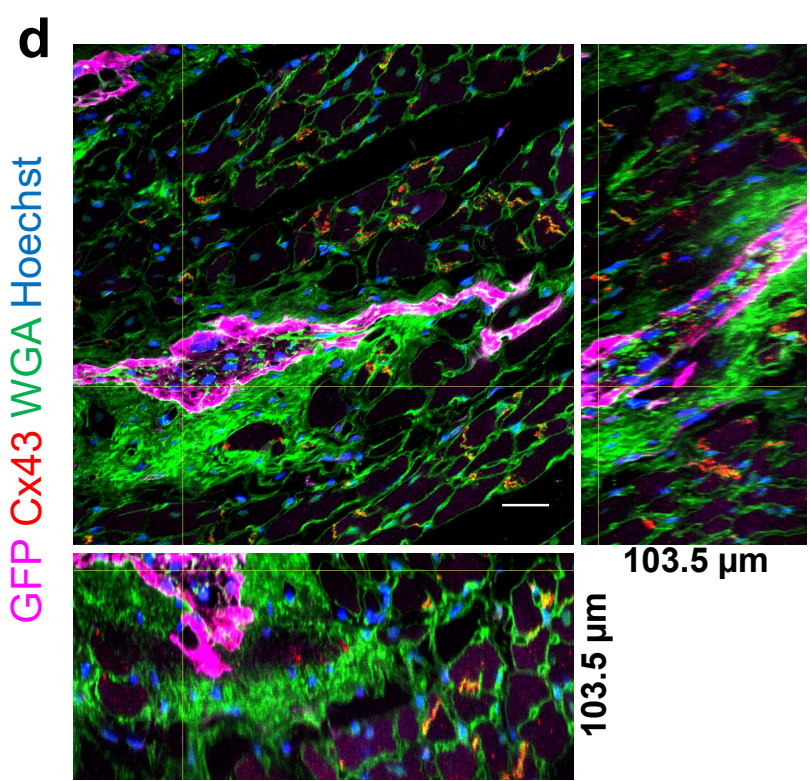

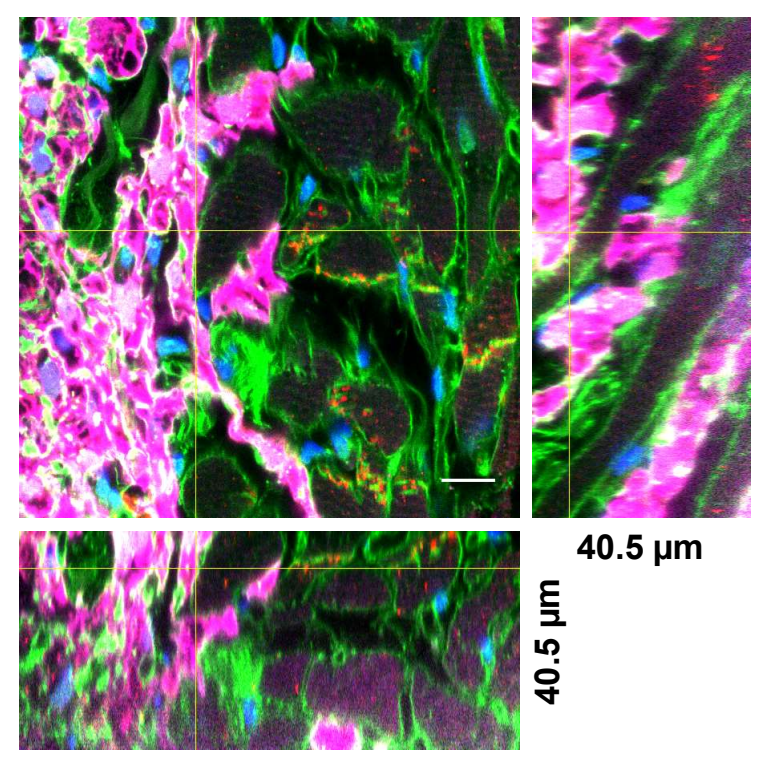

Figure 4 

not certified by peer review) is the author/funder. All rights reserved. No reuse allowed without permission.

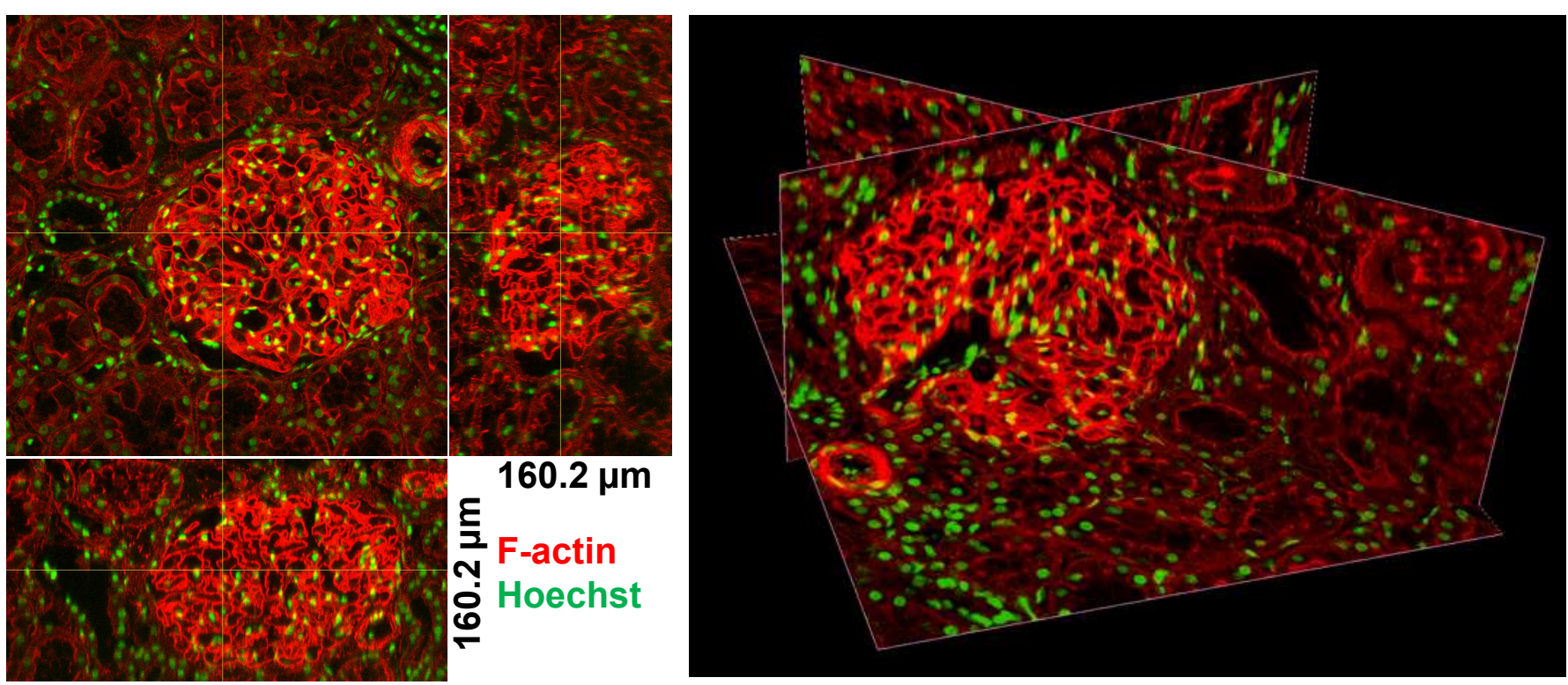

b
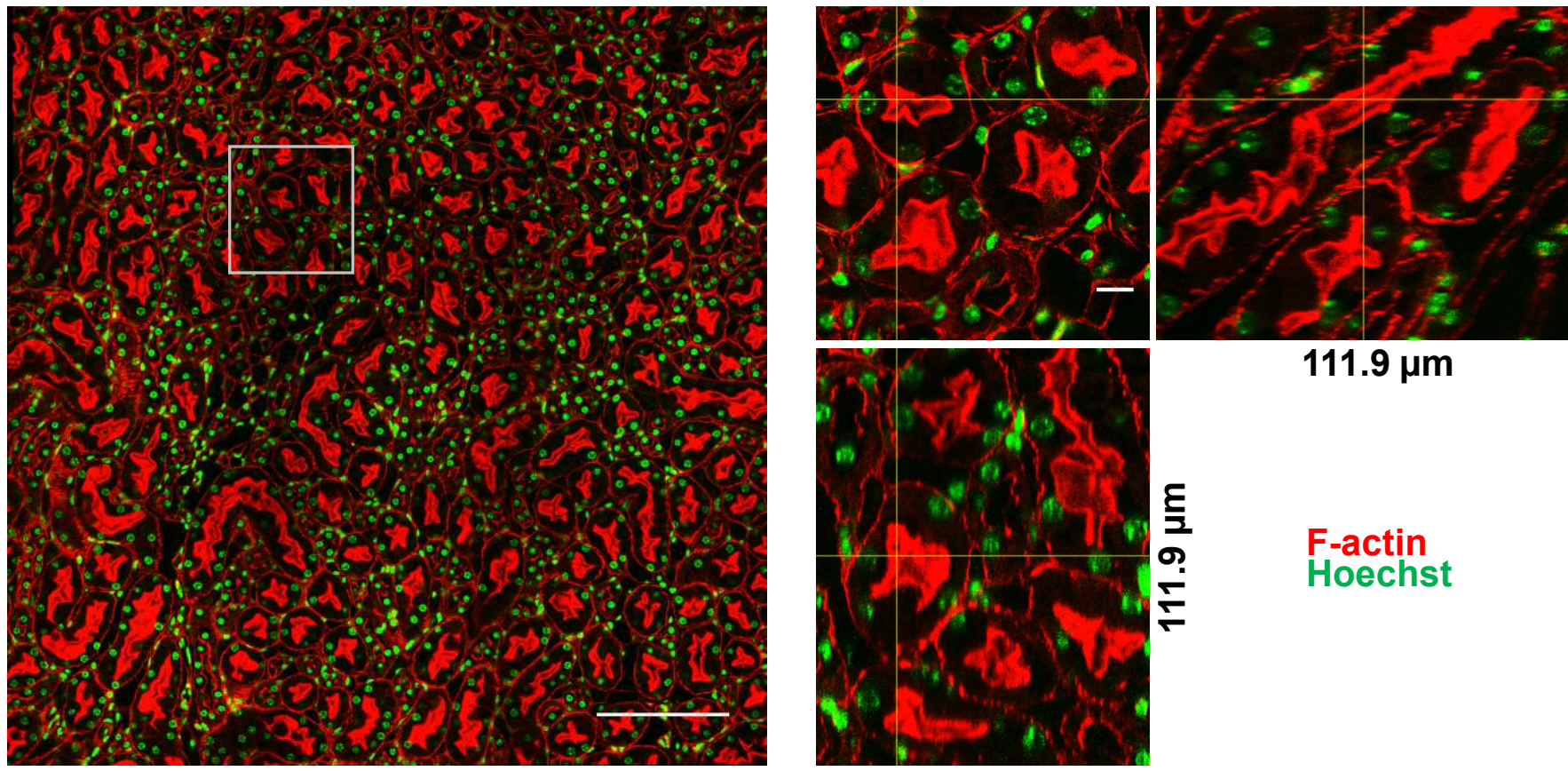

$111.9 \mu \mathrm{m}$

\section{F-actin}

Hoechst 
a

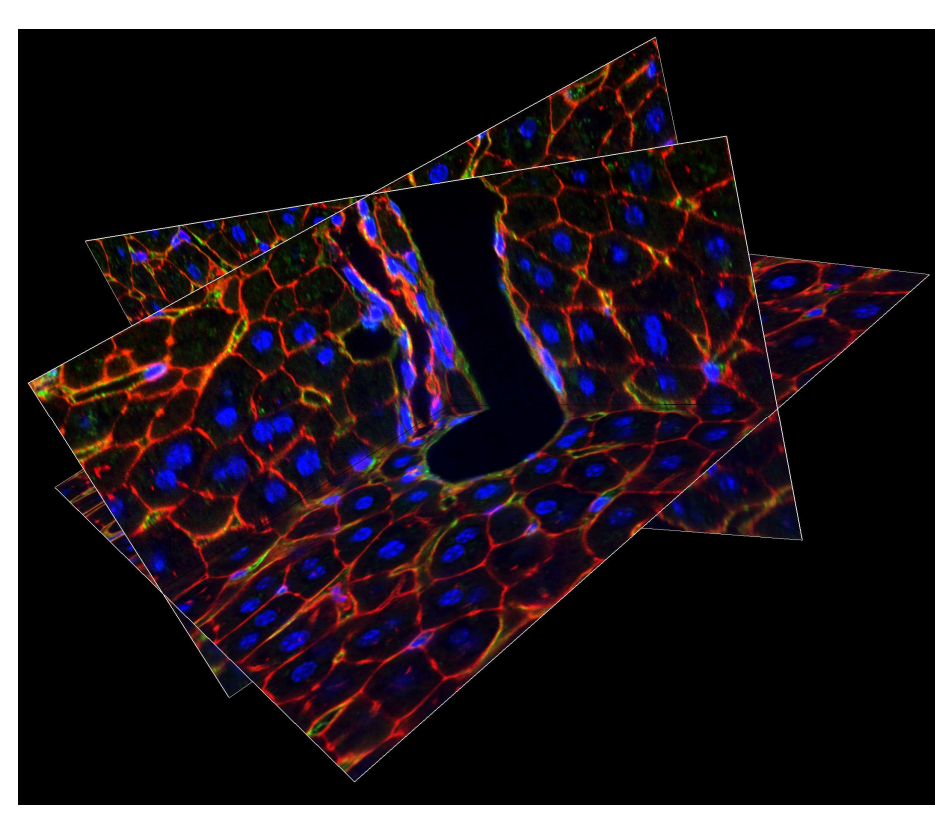

b

C

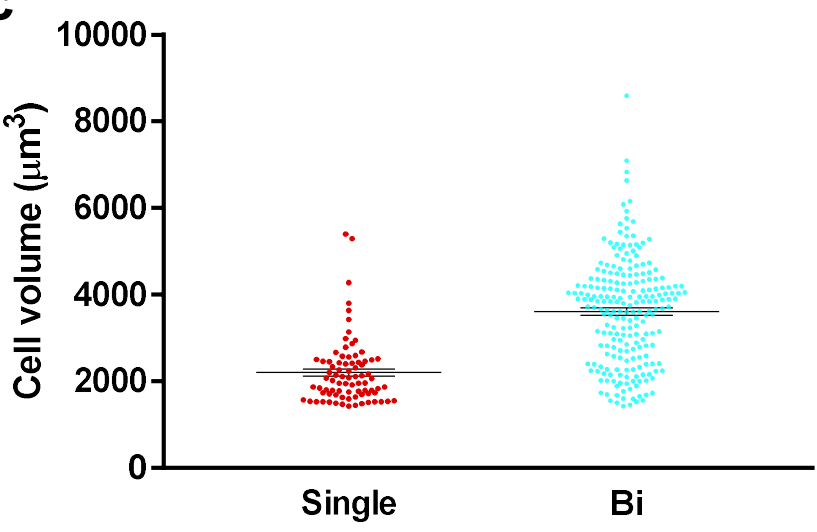

d

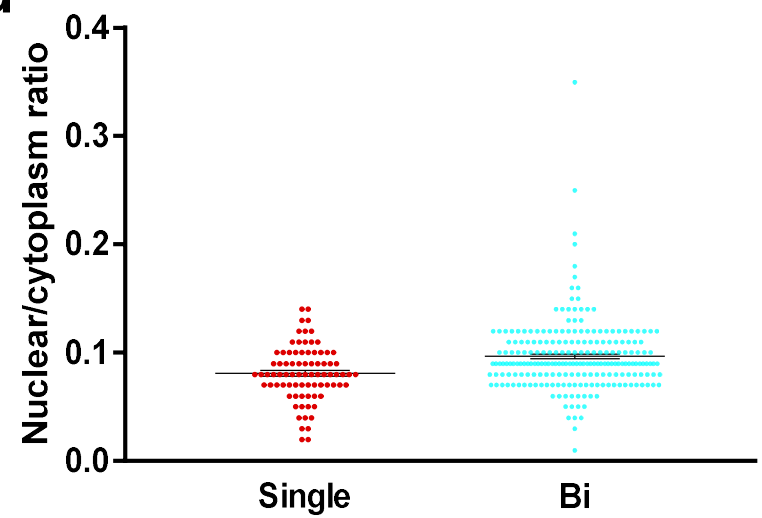


至quigment

$50 \mathrm{~mL}$ tube rotator

Orbital shaker

Vibratome (Leica VT1200s)

*Vibratome blade (TED Pella, 121-9)

*Scalpel or surgical scissors

Sharps waste container

Confocal microscope (Nikon A1R)

PC with image analysis software (ImageJ/FIJI, Nikon elements, or BitPlane Imaris)

${ }^{*}$ Hot plate

Painbrush, soft, synthetic bristles, \#0.

Blunt Forceps

Luer Lok syringe (optional, for perfusion)

Blunt canula (optional, for perfusion)

Canula clip (optional, for perfusion)

Suture (optional, for perfusion)

Cryomold

Lab tissue paper (i.e. Kimwipes) or gauss

Cotton swab

Nitrile gloves

Polypropylene tubes $(1.5 \mathrm{~mL}, 5 \mathrm{~mL})$

Nylon Mesh or Cell Strainer

24 well plates

Falcon tubes $(50 \mathrm{~mL})$

Sample baskets $(5 \mathrm{~mL}$ tube + cell strainers/mesh)

Petri dishes

Coverslips (\#0)

$100 \mathrm{~mL}$ Glass Jars

Coverslip caddy

Slides

Adhesive, solvent-resistant foil (Nashua Foil Tape, $279 \mu$ m thickness, catalog\# 1207802)

Reagents

Phosphate buffered saline (PBS) (Gibco catalog \# 21300-58)

KB buffer (mmol/L: KCl 20, KH2PO4 10, K+-glutamate 70, MgCl2 1, glucose 25, taurine 20, EGTA 0.5,

HEPES $10,0.1 \%$ bovine serum albumin, $\mathrm{pH}$ adjusted to 7.4 with $\mathrm{KOH}$ )

*16\% paraformaldehyde, ampoule in nitrogen. (Alfa Aesar catalog \# 43368)

*Methanol (Sigma catalog\# 34860)

Low melt agarose (Fisher Scientific catalog \# BP165-25)

Bovine Serum Albumin (BSA)

*Dimethyl Sulfoxide (DMSO)

*Sodium Azide

*Super glue

Normal Donkey Serum

*Triton X-100

Fluorescent beads (Fluorsbrite, Thermofisher, Sigma)

Primary antibodies: phosphorylated serine 10 histone $\mathrm{H} 3$ ( $\mathrm{pH} 3$, Abcam ab5176), trimethyl lysine 9 histone H3 (H3K9me3, Active Motif 39161), Heterochromatin Protein 1 gamma (HP1g, Active Motif 39981), Connexin 43 (Cx43) (Zymed catalog \# 71-0700), GFP (Novus catalog \# NB100-1770).

Secondary antibodies: (Alexa Fluor 488, 555, and 647, Thermo Fisher)

Chemical dyes: Wheat Germ Agglutinin (WGA) Alexa Fluor-647, WGA-Oregon Green, phalloidin-Alexa

Fluor 647, phalloidin-rhodamine, Hoechst 33342

Poly-L-lysine

*Isopropanol

*Benzyl alcohol

*Benzyl benzoate

Nail polish (Sally Hanson Insta-dry, clear) 
Sample comes out of agarose block during section

Morphology artifacts

Thickness of section not accurate

Low signal (broadly)

Low signal (interior/middle of section)

Distortions in z-dimension (one-sided)

Distortions in z-dimension (broadly) objective

Computer crashes

\section{Problem}

Solution

Work quickly with the molten agarose, blot the tissue gently but thoroughly, swirl the tissue in the molten agarose so that any residual PBS is mixed in with the agarose. Ensure there is enough agarose surrounding the tissue after trimming the block and that the block remains cold during sectioning. Use soft brushes, careful handling, slower vibratome blade advancing. Adjust fixation times. Adjust agarose \% for embedding.

We confirmed the vibratome section thickness is calibrated using Leica Vibrocheck. If the user does not have access to a vibratome calibrator, they can check the thickness by performing z-stacks on a confocal microscope and adjust the vibratome settings to compensate for differences in visualized thickness versus expected thickness. When using confocal imaging to assess section thickness, it is essential that the user imaging configuration has been validated with fluorescent bead standards

Use cold PFA and fix for no longer than 3-6 hours. Ensure $\mathrm{MeOH}$ is not warming up during post-fix. Keep isopropanol incubations at 50 seconds each, extending this step will reduce signal. Use fresh samples or store samples in accordance with the protocol notes to prevent signal decay.

Add 1\% DMSO to staining buffer, increase rocker speed, perform Ab staining steps at room temp, or extend incubation time. Increase staining reagent concentration. Though not suitable for studies quantifying signal intensity, uneven staining across the thickness can be overcome using adaptive imaging where excitation laser power is adjusted throughout the acquisition to give uniform signal, or post processing algorithms, such as ImageJ/FIJI (Menu: Images $\rightarrow$ Adjust $\rightarrow$ Bleach Correction $\rightarrow$ Histogram Matching).

Adjust correction collar on objective lens or adjust coverslip thickness and confirm with fluorescent beads; or (not recommended for quantitative studies) correct z-step size post processing using calibration values determine by fluorescent microsphere standards.

This is consistent with going over the working distance limits of the objective, where the objective begins pushing up against the coverslip and z-step size is no longer accurate. This can also potentially damage the objective. Use thinner coverslips (i.e. \#0) or switch from a $60 x$ oil objective to $40 \mathrm{x}$ oil for more working distance.

Use thin \#0 coverslips. Ensure that the sample is flat and well-adhered on the coverslip throughout the clearing steps, taking care to transfer the Sample cannot get in focus on high powersamples gently from each solution. Gently handle the slides after sealing with nail polish. Though the sample is sealed between the coverslip and slide, if it gets detached from the coverslip this will result in less sample imageable within the working distance of the objective.

Reduce bit-depth, the human eye cannot distinguish more than 250 shades of gray, thus even 8-bit images are suitable for most applications. Alternatively, sub-sample regions of interest. Commercial software, such as Nikon Elements or Bitplane Imaris, can handle large file sizes more efficiently. 